Bull. Korean Math. Soc. 52 (2015), No. 3, pp. 895-906

http://dx.doi.org/10.4134/BKMS.2015.52.3.895

\title{
THE ULTIMATE RUIN PROBABILITY OF A DEPENDENT DELAYED-CLAIM RISK MODEL PERTURBED BY DIFFUSION WITH CONSTANT FORCE OF INTEREST
}

\author{
Qingwu Gao, Erli Zhang, and Na Jin
}

\begin{abstract}
Recently, Li [12] gave an asymptotic formula for the ultimate ruin probability in a delayed-claim risk model with constant force of interest and pairwise quasi-asymptotically independent and extendedregularly-varying-tailed claims. This paper extends Li's result to the case in which the risk model is perturbed by diffusion, the claims are consistently-varying-tailed and the main-claim interarrival times are widely lower orthant dependent.
\end{abstract}

\section{Introduction}

Consider a delayed-claim risk model perturbed by diffusion with constant force of interest, which involves two kinds of insurance claims, namely the main claims and the by-claims, such that each main claim may cause a byclaim occurring after a period of delay. In this model, the main claims $\left\{X_{i}, i \geq\right.$ $1\}$, by-claims $\left\{Y_{i}, i \geq 1\right\}$, inter-arrival times of main claims $\left\{\theta_{i}, i \geq 1\right\}$ are three sequences of nonnegative and identically distributed, but not necessarily independent, random variables (r.v.s) with common distributions $F, G$ and $K$, respectively. Denote by $\tau_{i}=\sum_{k=1}^{i} \theta_{k}, i \geq 1$, the arrival times of successive main claims, which constitute a counting process

$$
N(t)=\sup \left\{i \geq 1: \tau_{i} \leq t\right\}, \quad t \geq 0
$$

and by $\left\{T_{i}, i \geq 1\right\}$ the delay times of by-claims, which are nonnegative (but possibly degenerated at 0 ), arbitrarily dependent and identically distributed r.v.s with common distribution $H$.

Assume that the total amount of premiums accumulated before time $t \geq 0$, denoted by $C(t)$, is a nonnegative and nondecreasing stochastic process with $C(0)=0$ and $C(t)<\infty$ almost surely for every $0 \leq t<\infty$; and that the diffusion process, as a perturbed term, $\{B(t), t \geq 0\}$ is a standard Brownian

Received March 30, 2014; Revised September 19, 2014.

2010 Mathematics Subject Classification. Primary 62E20; Secondary 62P05, 91B30.

Key words and phrases. asymptotic ruin probability, delayed claim, diffusion, pairwise quasi-asymptotic independence, widely lower orthant dependence. 
motion with volatility parameter $\sigma \geq 0$ and independent of the other sources of randomness. In practice, the diffusion-perturbed term can be interpreted as an additional uncertainty of the aggregate claims or the premium income of an insurance company. Let $r>0$ be the constant force of interest and $x \geq 0$ be the insurer's initial reserve. Then the total reserve up to time $t \geq 0$, denoted by $R(t)$, satisfies

$$
\begin{aligned}
R(t)= & x e^{r t}+\int_{0-}^{t} e^{r(t-s)} d C(s)-\sum_{i=1}^{\infty} X_{i} e^{r\left(t-\tau_{i}\right)} \mathbf{1}_{\left\{\tau_{i} \leq t\right\}} \\
& -\sum_{i=1}^{\infty} Y_{i} e^{r\left(t-\tau_{i}-T_{i}\right)} \mathbf{1}_{\left\{\tau_{i}+T_{i} \leq t\right\}}+\sigma \int_{0-}^{t} e^{r(t-s)} d B(s),
\end{aligned}
$$

where $\mathbf{1}_{E}$ is the indicator function of an event $E$. Hence the ultimate ruin probability is defined by

$$
\psi_{r}(x)=P(R(t)<0 \text { for some } t \geq 0) .
$$

It is well-known that the delayed-claim risk model was firstly introduced by Waters and Papatriandafylou [22] so that the independence assumption between claim sizes and their inter-arrival times can be relaxed, and since then it has been extensively investigated by many researchers. See, for example, Yuen and Guo [26], Xiao and Guo [23], Li and Wu [13], among others. For the continuous-time counterparts, the readers are referred to Yuen et al. [27], Xie and Zou [24, 25], Meng and Wang [15], Zou and Xie [28], and references therein. We notice that all the references above only discussed the case when the claims are light-tailed and mutually independent. But recently, Li [12] considered the delayed-claim risk model with heavy-tailed and dependent claims, and an asymptotic formula for the ultimate ruin probability was reached. So in the following, we introduce some dependence structures and some classes of heavytailed distributions.

Wang et al. [20] introduced the widely lower orthant dependent (WLOD) structure. Say that r.v.s $\left\{\xi_{i}, i \geq 1\right\}$ are WLOD, if there exists a sequence of finite positive numbers $\left\{g_{L}(n), n \geq 1\right\}$ such that for each $n \geq 1$ and all $x_{i} \in(-\infty, \infty), 1 \leq i \leq n$,

$$
P\left(\bigcap_{i=1}^{n}\left\{\xi_{i} \leq x_{i}\right\}\right) \leq g_{L}(n) \prod_{i=1}^{n} P\left(\xi_{i} \leq x_{i}\right) .
$$

Clearly, if $\left\{\xi_{i}, i \geq 1\right\}$ are WLOD r.v.s, then for each $n \geq 1$ and any $s>0$,

$$
E \exp \left\{-s \sum_{i=1}^{n} \xi_{i}\right\} \leq g_{L}(n) \prod_{i=1}^{n} E e^{-s \xi_{i}} .
$$

Also, Chen and Yuen [2] proposed a more general dependence structure below. Say that r.v.s $\left\{\xi_{i}, i \geq 1\right\}$ are pairwise quasi-asymptotically independent 
(PQAI), if for any $1 \leq i \neq j<\infty$,

$$
\lim _{x \rightarrow \infty} P\left(\left|\xi_{i}\right| \wedge \xi_{j}>x \mid \xi_{i} \vee \xi_{j}>x\right)=0,
$$

where we denote $x \wedge y=\min \{x, y\}$ and $x \vee y=\max \{x, y\}$. For further study on the above dependence structures and their analogues, we refer to Geluk and Tang [11], Wang and Cheng [21], Liu et al. [14], Gao and Jin [6], Gao and Liu [8], Gao and Yang [9, 10], Gao et al. [7], Gao and Bao [5], and others.

Henceforth, all limit relationships are for $x \rightarrow \infty$ unless mentioned otherwise. For two positive functions $a(\cdot)$ and $b(\cdot)$, we write $a(x)=O(1) b(x)$ if $\limsup a(x) / b(x)=C<\infty$, write $a(x)=o(1) b(x)$ if $C=0$, write $a(x) \lesssim b(x)$ or $b(x) \gtrsim a(x)$ if $C \leq 1$, write $a(x) \sim b(x)$ if $a(x) \lesssim b(x)$ and $b(x) \lesssim a(x)$, write $a(x) \asymp b(x)$ if $a(x)=O(1) b(x)$ and $b(x)=O(1) a(x)$.

In the paper, we are concerned with some classes of heavy-tailed distributions, which have no finite exponential moments. Say that a r.v. $\xi$ or its distribution $V$ belongs to the ERV class of extended-regularly-varyingtailed distributions if there exist some $0<\alpha \leq \beta<\infty$ such that $y^{-\beta} \leq$ $\bar{V}_{*}(y) \leq \bar{V}^{*}(y) \leq y^{-\alpha}$ for all $y \geq 1$, where $\bar{V}_{*}(y)=\liminf \bar{V}(x y) / \bar{V}(x)$ and $\bar{V}^{*}(y)=\lim \sup \bar{V}(x y) / \bar{V}(x)$; belongs to the consistently-varying-tailed class, denoted by $V \in \mathcal{C}$, if $\lim _{y \searrow 1} \bar{V}_{*}(y)=1$ or $\lim _{y} \nearrow_{1} \bar{V}^{*}(y)=1$; belongs to the dominatedly-varying-tailed class, denoted by $V \in \mathcal{D}$, if $\bar{V}^{*}(y)<\infty$ for all $y>0$; belongs to the long-tailed class, denoted by $V \in \mathcal{L}$, if

$$
\bar{V}(x+y) \sim \bar{V}(x) \text { for all } y>0
$$

In conclusion,

$$
E R V \subset \mathcal{C} \subset \mathcal{L} \cap \mathcal{D} \text {. }
$$

More details of heavy-tailed distributions and their applications can be found in Bingham et al. [1] and Embrechts et al. [4].

Recently, Li [12] showed that in the delayed-claim risk model (1.1) with $\sigma=0$ and $C(\cdot)$ a deterministic linear function, if the claim sizes $\left\{X_{i}, Y_{i}, i \geq 1\right\}$ are PQAI, random pairs $\left\{\left(X_{i}, Y_{i}\right), i \geq 1\right\}$ are identically distributed with marginal distributions $F \in E R V$ and $G \in E R V$, the inter-arrival times of main claims $\left\{\theta_{i}, i \geq 1\right\}$ are independent, and $\left\{X_{i}, Y_{i}, i \geq 1\right\},\left\{\theta_{i}, i \geq 1\right\}$ and $\left\{T_{i}, i \geq 1\right\}$ are mutually independent, then

$$
\psi_{r}(x) \sim \int_{0-}^{\infty} \bar{F}\left(x e^{r t}\right) d E N(t)+\int_{0-}^{\infty} \int_{0-}^{\infty} \bar{G}\left(x e^{r(u+t)}\right) d H(u) d E N(t) .
$$

Motivated by Li's result in [12], in the paper we further consider the delayedclaim risk model (1.1) perturbed by diffusion, in which the claim sizes (including main claims and by-claims) are PQAI and consistently-varying-tailed, the interarrival times of main claims satisfy WLOD structure, and the premium income follows a general stochastic process. In our main result, we will discuss two cases, one is that the premium process $\{C(t), t \geq 0\}$ is independent of the other sources of randomness, and the other is that $\{C(t), t \geq 0\}$ is not necessarily so. 
In the rest part of this paper, we will present the main result in Section 2, and prove it in Section 3 after preparing some lemmas.

\section{Main result}

In this section, we state our main result. For a distribution $V$ and any $y>0$, we set

$$
J_{V}^{+}=-\lim _{y \rightarrow \infty} \log \bar{V}_{*}(y) / \log y \quad \text { and } \quad J_{V}^{-}=-\lim _{y \rightarrow \infty} \log \bar{V}^{*}(y) / \log y .
$$

Assume that the total discounted amount of premiums is finite, namely,

$$
0 \leq \widetilde{C}=\int_{0-}^{\infty} e^{-r s} d C(s)<\infty \quad \text { almost surely } .
$$

Our main result is given below.

Theorem 2.1. Consider the delayed-claim risk model (1.1), in which the claim sizes $\left\{X_{i}, Y_{i}, i \geq 1\right\}$ are PQAI r.v.s, random pairs $\left\{\left(X_{i}, Y_{i}\right), i \geq 1\right\}$ are identically distributed by marginal distributions $F \in \mathcal{C}$ and $G \in \mathcal{C}$ with $J_{F}^{-}>0$ and $J_{G}^{-}>0$, respectively, and $\left\{X_{i}, Y_{i}, i \geq 1\right\},\left\{\theta_{i}, i \geq 1\right\}$ and $\left\{T_{i}, i \geq 1\right\}$ are mutually independent. Assume that the inter-arrival times of main claims $\left\{\theta_{i}, i \geq 1\right\}$ are WLOD r.v.s such that

$$
\lim _{n \rightarrow \infty} g_{L}(n) e^{-\epsilon n}=0
$$

holds for every $\epsilon>0$, depending on $F, G, K$ and $H$. Then relation (1.5) holds, if one of the following conditions is true:

1. the premium process $\{C(t), t \geq 0\}$ is independent of the other sources of randomness;

2. the total discounted amount of premiums satisfies

$$
P(\widetilde{C}>x)=o(1)(\bar{F}(x)+\bar{G}(x)) .
$$

Remark 2.1. As was pointed out by Tang [16], condition (2.3), which does not require the independence between the premium process and the other sources of randomness, allows for a more realistic case that the premium income varies as a deterministic or stochastic function of the insurer's current reserve.

\section{Proof of Theorem 2.1}

Before proving Theorem 2.1, we prepare the following lemmas. The first one is a direct result of Proposition 2.2.1 of Bingham et al. [1] or Lemma 3.5 of Tang and Tsitsiashvili [17].

Lemma 3.1. If a distribution $V \in \mathcal{D}$ with $J_{V}^{-}>0$, then for any $0<\hat{p}<J_{V}^{-} \leq$ $J_{V}^{+}<p<\infty$, there exist some $C>1$ and $D>0$ such that

$$
C^{-1}\left(\frac{x}{y}\right)^{\hat{p}} \leq \frac{\bar{V}(y)}{\bar{V}(x)} \leq C\left(\frac{x}{y}\right)^{p} \quad \text { for all } x \geq y \geq D \text {. }
$$


The second lemma is from Theorem 3.3(iv) of Cline and Samorodnitsky [3] and Lemma 2.5 of Wang et al. [19].

Lemma 3.2. Let $\xi$ and $\eta$ be two independent r.v.s, where $\xi$ is distributed by $V$, and $\eta$ is nonnegative, not degenerate at 0 and satisfying $E \eta^{p}<\infty$ for some $p>J_{V}^{+}$.

(1) If $V \in \mathcal{D}$, then $P(\xi \eta>x) \asymp \bar{V}(x)$.

(2) If $V \in \mathcal{C}$, then the distribution of $\xi \eta$ still belongs to the class $\mathcal{C}$.

The third lemma is a restatement of Theorem 2.1 of Li [12]. Also, see Lemma 3.2 of Gao and Yang [10]. It should be mentioned that the asymptotic formula in the lemma was first developed by Tang and Tsitsiashvili [18].

Lemma 3.3. If $\left\{\xi_{i}, 1 \leq i \leq n\right\}$ are $n$ PQAI and real-valued r.v.s with distributions $V_{i} \in \mathcal{C}, 1 \leq i \leq n$, respectively, then for any fixed $0<a \leq b<\infty$,

$$
P\left(\sum_{i=1}^{n} c_{i} \xi_{i}>x\right) \sim \sum_{i=1}^{n} \bar{V}_{i}\left(\frac{x}{c_{i}}\right)
$$

holds uniformly for all $\left(c_{1}, c_{2}, \ldots, c_{n}\right) \in[a, b]^{n}$.

The lemma below is due to Lemma 3.1 of Chen and Yuen [2] or Theorem 2.2 of $\mathrm{Li}[12]$.

Lemma 3.4. Let $\left\{\xi_{i}, 1 \leq i \leq n\right\}$ be $n P Q A I$ and real-valued r.v.s with distributions $V_{i} \in \mathcal{D}, 1 \leq i \leq n$, respectively, and $\left\{\eta_{i}, 1 \leq i \leq n\right\}$ be $n$ nonnegative r.v.s, independent of $\left\{\xi_{i}, \overline{1} \leq i \leq n\right\}$ and satisfying $E \overline{\eta_{i}^{p}}<\infty$ for some $p>\bigvee_{i=1}^{n} J_{V_{i}}^{+}$, $1 \leq i \leq n$. Then $\left\{\xi_{i} \eta_{i}, 1 \leq i \leq n\right\}$ are still PQAI.

Lemma 3.5. If $\left\{\xi_{i}, \eta_{i}, i \geq 1\right\}$ are PQAI and real-valued r.v.s with $\xi_{i}$ and $\eta_{i}$ distributed by $V_{i} \in \mathcal{C}$ and $W_{i} \in \mathcal{C}, i \geq 1$, respectively, then $\left\{\zeta_{i}=\xi_{i}+\eta_{i}, i \geq 1\right\}$ are still PQAI r.v.s with distributions $U_{i} \in \mathcal{C}, i \geq 1$.

Proof. By Theorem 2.5(ii) of Li ([12]), we know that $\left\{\zeta_{i}, i \geq 1\right\}$ are PQAI. By Lemma 3.3, it holds that

$$
\bar{U}_{i}(x) \sim \bar{V}_{i}(x)+\bar{W}_{i}(x), \quad i \geq 1
$$

which, along with $V_{i} \in \mathcal{C}$ and $W_{i} \in \mathcal{C}, i \geq 1$, leads to $U_{i} \in \mathcal{C}, i \geq 1$.

The last lemma comes from Lemma 3.3 of Gao et al. [7].

Lemma 3.6. Consider the main-claim arrival process $\{N(t), t \geq 0\}$ with $W L O D$ inter-arrival times $\left\{\theta_{i}, i \geq 1\right\}$ satisfying $(2.2)$ for every $\epsilon>0$. Then for any fixed $t>0$ and any $p>0$,

$$
E(N(t))^{p}<\infty .
$$

Now we proceed to prove Theorem 2.1. 
Proof of Theorem 2.1. By the reserve process (1.1), we get its discounted value as

$$
\begin{aligned}
\widetilde{R}(t)= & x+\int_{0-}^{t} e^{-r s} d C(s)-\sum_{i=1}^{\infty} X_{i} e^{-r \tau_{i}} \mathbf{1}_{\left\{\tau_{i} \leq t\right\}} \\
& -\sum_{i=1}^{\infty} Y_{i} e^{-r\left(\tau_{i}+T_{i}\right)} \mathbf{1}_{\left\{\tau_{i}+T_{i} \leq t\right\}}+\sigma \int_{0-}^{t} e^{-r s} d B(s), \quad t \geq 0 .
\end{aligned}
$$

Then by (1.2), it follows that

$$
\psi_{r}(x)=P(\widetilde{R}(t)<0 \text { for some } t \geq 0) .
$$

Let $\widetilde{B}=\sigma \sup _{t \in[0, \infty]}\left|\int_{0-}^{t} e^{-r s} d B(s)\right|$. It is well-known that the stochastic integral $\int_{0-}^{t} e^{-r s} d B(s), 0<t \leq \infty$, follows a normal distribution with mean 0 and variance $\int_{0}^{t} e^{-2 r s} d s$, then by many classic martingale inequalities, $\widetilde{B}$ has finite moments of arbitrary orders. So if a distribution $U \in \mathcal{D}$, then

$$
P(\widetilde{B}>x)=o(1) \bar{U}(x) .
$$

Clearly, by (3.2) we get

$$
\widetilde{R}(t) \geq x-\sum_{i=1}^{\infty}\left(X_{i}+Y_{i} e^{-r T_{i}}\right) e^{-r \tau_{i}}-\widetilde{B}, \quad t \geq 0,
$$

which, along with (3.3), implies that

$$
\psi_{r}(x) \leq P\left(\sum_{i=1}^{\infty}\left(X_{i}+Y_{i} e^{-r T_{i}}\right) e^{-r \tau_{i}}+\widetilde{B}>x\right) .
$$

On the other hand, again by (3.2) we have

$$
\widetilde{R}(t) \leq x+\widetilde{C}-D_{r}(t)+\widetilde{B}, \quad t \geq 0,
$$

where $D_{r}(t)=\sum_{i=1}^{\infty} X_{i} e^{-r \tau_{i}} \mathbf{1}_{\left\{\tau_{i} \leq t\right\}}+\sum_{i=1}^{\infty} Y_{i} e^{-r\left(\tau_{i}+T_{i}\right)} \mathbf{1}_{\left\{\tau_{i}+T_{i} \leq t\right\}}$, and $\widetilde{C}$ is defined in (2.1). Hence by (3.3), we derive that

$$
\begin{aligned}
\psi_{r}(x) & \geq P\left(D_{r}(t)-\widetilde{B}>x+\widetilde{C} \quad \text { for some } t \geq 0\right) \\
& =P\left(\bigcup_{t \geq 0}\left\{D_{r}(t)-\widetilde{B}>x+\widetilde{C}\right\}\right) \\
& \geq P\left(D_{r}(\infty)-\widetilde{B}>x+\widetilde{C}\right) \\
& =P\left(\sum_{i=1}^{\infty}\left(X_{i}+Y_{i} e^{-r T_{i}}\right) e^{-r \tau_{i}}-\widetilde{B}>x+\widetilde{C}\right) .
\end{aligned}
$$

Firstly, we deal with the asymptotic upper-bound of $\psi_{r}(x)$. By Lemmas $3.2(2)$ and 3.4, one can easily see that the common distribution of $Y_{i} e^{-r T_{i}}$, $i \geq 1$, belongs to the class $\mathcal{C}$, and $\left\{X_{i}, Y_{i} e^{-r T_{i}}, i \geq 1\right\}$ are PQAI. Then by 
Lemma 3.5, we know that $X_{i}+Y_{i} e^{-r T_{i}}, i \geq 1$, are also PQAI and identically distributed by $U \in \mathcal{C}$. So, following the proof of Lemma 3.5 of Gao and Liu [8] or going along the similar lines of the proof of Lemma 3.5 of Gao and Jin [6], there exists a positive integer $n_{0}$ such that for any $0<v<1$,

$$
\begin{aligned}
& P\left(\sum_{i=n_{0}+1}^{\infty}\left(X_{i}+Y_{i} e^{-r T_{i}}\right) e^{-r \tau_{i}}>\frac{v x}{2}\right) \\
= & o(1) P\left(\left(X_{1}+Y_{1} e^{-r T_{1}}\right) e^{-r \tau_{1}}>x\right) .
\end{aligned}
$$

Note that the common distribution of $X_{i}+Y_{i} e^{-r T_{i}}, i \geq 1$, belongs to $\mathcal{C}$, then again by Lemma $3.2(2)$, the distributions of $\left(X_{i}+Y_{i} e^{-r T_{i}}\right) e^{-r \tau_{i}}, i \geq 1$, also belong to $\mathcal{C}$. Hence for any given $\varepsilon>0$, there exists a number $v_{0}, 0<v_{0}<1$, such that for all large $x$,

$$
\begin{array}{r}
\sum_{i=1}^{n_{0}} P\left(\left(X_{i}+Y_{i} e^{-r T_{i}}\right) e^{-r \tau_{i}}>\left(1-v_{0}\right) x\right) \\
\leq(1+\varepsilon) \sum_{i=1}^{n_{0}} P\left(\left(X_{i}+Y_{i} e^{-r T_{i}}\right) e^{-r \tau_{i}}>x\right) .
\end{array}
$$

Let $n_{0}$ and $v_{0}$ be fixed as above. By (3.5), we have

$$
\begin{aligned}
\psi_{r}(x) \leq & P\left(\sum_{i=1}^{n_{0}}\left(X_{i}+Y_{i} e^{-r T_{i}}\right) e^{-r \tau_{i}}>\left(1-v_{0}\right) x\right) \\
& +P\left(\sum_{i=n_{0}+1}^{\infty}\left(X_{i}+Y_{i} e^{-r T_{i}}\right) e^{-r \tau_{i}}>\frac{v_{0} x}{2}\right)+P\left(\widetilde{B}>\frac{v_{0} x}{2}\right) \\
= & \sum_{i=1}^{3} H_{i} .
\end{aligned}
$$

For $H_{1}$, by Theorem 3.2 of Chen and Yuen [2] and (3.8), we show that for all large $x$,

$$
\begin{aligned}
H_{1} & \sim \sum_{i=1}^{n_{0}} P\left(\left(X_{i}+Y_{i} e^{-r T_{i}}\right) e^{-r \tau_{i}}>\left(1-v_{0}\right) x\right) \\
& \leq(1+\varepsilon) \sum_{i=1}^{n_{0}} P\left(\left(X_{i}+Y_{i} e^{-r T_{i}}\right) e^{-r \tau_{i}}>x\right) .
\end{aligned}
$$

For $\mathrm{H}_{2}$, by (3.7) with $v=v_{0}$, we get

$$
H_{2}=o(1) P\left(\left(X_{1}+Y_{1} e^{-r T_{1}}\right) e^{-r \tau_{1}}>x\right) .
$$

For $H_{3}$, by (3.4), $U \in \mathcal{C} \subset \mathcal{D}$ and Lemma 3.2(1), we obtain

$$
H_{3}=o(1)\left(\left(X_{1}+Y_{1} e^{-r T_{1}}\right) e^{-r \tau_{1}}>x\right) \text {. }
$$


Therefore, substituting the derivations for $H_{i}, i=1,2,3$, into (3.9) and considering the arbitrariness of $\varepsilon>0$ can imply that

$$
\begin{aligned}
\psi_{r}(x) & \lesssim \sum_{i=1}^{\infty} P\left(\left(X_{i}+Y_{i} e^{-r T_{i}}\right) e^{-r \tau_{i}}>x\right) \\
& \sim \int_{0-}^{\infty} \bar{F}\left(x e^{r t}\right) d E N(t)+\int_{0-}^{\infty} \int_{0-}^{\infty} \bar{G}\left(x e^{r(u+t)}\right) d H(u) d E N(t),
\end{aligned}
$$

where in the second step we used Lemma 3.3.

Subsequently, we turn to the asymptotic lower-bound of $\psi_{r}(x)$. As mentioned above, $X_{i}+Y_{i} e^{-r T_{i}}, i \geq 1$, are identically distributed by $U \in \mathcal{C} \subset \mathcal{D}$. Thus by (3.1), it holds that for all $x \geq D$ and any $0<t<\infty$,

$$
\frac{\int_{t}^{\infty} \bar{U}\left(x e^{r s}\right) d E N(s)}{\int_{0-}^{\infty} \bar{U}\left(x e^{r s}\right) d E N(s)}=\frac{\int_{t}^{\infty} \bar{U}\left(x e^{r s}\right) / \bar{U}(x) d E N(s)}{\int_{0-}^{\infty} \bar{U}\left(x e^{r s}\right) / \bar{U}(x) d E N(s)} \leq C^{2} \frac{\int_{t}^{\infty} e^{-r \hat{p} s} d E N(s)}{\int_{0-}^{\infty} e^{-r p s} d E N(s)}
$$

By (1.3), we get

$$
\int_{0-}^{\infty} e^{-r p s} d E N(s)=\sum_{n=1}^{\infty} E\left(e^{-r p \tau_{n}}\right) \leq \sum_{n=1}^{\infty} g_{L}(n)\left(E e^{-r p \theta_{1}}\right)^{n} .
$$

Take $\epsilon=-\log \left(E e^{-r p \theta_{1}}\right)-c$ for some $c>0$ in $(2.2)$, then there exists a positive integer $n_{1}$ such that for all $n \geq n_{1}$,

$$
g_{L}(n) \leq e^{-c n} \exp \left\{-n \log \left(E e^{-r p \theta_{1}}\right)\right\}
$$

Thus, we have

$$
\int_{0-}^{\infty} e^{-r p s} d E N(s) \leq \sum_{n=1}^{n_{1}-1} g_{L}(n)\left(E e^{-r p \theta_{1}}\right)^{n}+\sum_{n=n_{1}}^{\infty} e^{-c n}<\infty .
$$

Similarly,

$$
\int_{0-}^{\infty} e^{-r \hat{p} s} d E N(s)<\infty
$$

Hence, the third item of (3.11) tends to 0 as $t \rightarrow \infty$, which yields that for any given $\varepsilon>0$, there exists a number $t_{0}, 0<t_{0}<\infty$, such that for all $x \geq D$,

$$
\int_{t_{0}}^{\infty} \bar{U}\left(x e^{r t}\right) d E N(t) \leq \varepsilon \int_{0-}^{\infty} \bar{U}\left(x e^{r t}\right) d E N(t) .
$$

Write $\widetilde{D}_{r}\left(t_{0}\right)=\sum_{i=1}^{\infty}\left(X_{i}+Y_{i} e^{-r T_{i}}\right) e^{-r \tau_{i}} \mathbf{1}_{\left\{\tau_{i} \leq t_{0}\right\}}$, where $t_{0}$ is fixed as above. Now we estimate the asymptotic lower-bound of $P\left(\widetilde{D}_{r}\left(t_{0}\right)>x\right)$, which will be used in the proof below. For an arbitrarily fixed integer $m$, we derive from Lemma 3.3 that

$$
\begin{aligned}
& P\left(\widetilde{D}_{r}\left(t_{0}\right)>x\right) \\
\geq & \sum_{n=1}^{m} P\left(\sum_{i=1}^{n}\left(X_{i}+Y_{i} e^{-r T_{i}}\right) e^{-r \tau_{i}}>x, N\left(t_{0}\right)=n\right)
\end{aligned}
$$




$$
\begin{aligned}
= & \sum_{n=1}^{m} \int_{\left\{0<t_{1} \leq t_{2} \leq \cdots \leq t_{n} \leq t_{0}, t_{n+1}>t_{0}\right\}} P\left(\sum_{i=1}^{n}\left(X_{i}+Y_{i} e^{-r T_{i}}\right) e^{-r t_{i}}>x\right) \\
& d G\left(t_{1}, t_{2}, \ldots, t_{n+1}\right) \\
\sim & \left(\sum_{n=1}^{\infty}-\sum_{n=m+1}^{\infty}\right) \sum_{i=1}^{n} P\left(\left(X_{i}+Y_{i} e^{-r T_{i}}\right) e^{-r \tau_{i}}>x, N\left(t_{0}\right)=n\right) \\
= & \sum_{i=1}^{\infty} P\left(\left(X_{i}+Y_{i} e^{-r T_{i}}\right) e^{-r \tau_{i}} \mathbf{1}_{\left\{\tau_{i} \leq t_{0}\right\}}>x\right)-H_{4},
\end{aligned}
$$

where $G\left(t_{1}, t_{2}, \ldots, t_{n+1}\right)$ is the joint distribution of $\left(\tau_{1}, \tau_{2}, \ldots, \tau_{n+1}\right), 1 \leq n \leq$ $m$. For $\mathrm{H}_{4}$, it follows from Lemma 3.6 that

$$
\begin{aligned}
H_{4} & \leq \bar{U}(x) \sum_{n=m+1}^{\infty} n P\left(N\left(t_{0}\right)=n\right) \\
& =\bar{U}(x) E N\left(t_{0}\right) \mathbf{1}_{\left\{N\left(t_{0}\right)>m\right\}}=o(1) \bar{U}(x) \text { as } m \rightarrow \infty,
\end{aligned}
$$

which, along with $U \in \mathcal{C} \subset \mathcal{D}$ and Lemma 3.2(1), yields that

$$
H_{4}=o(1) P\left(\left(X_{1}+Y_{1} e^{-r T_{1}}\right) e^{-r \tau_{1}} \mathbf{1}_{\left\{\tau_{1} \leq t_{0}\right\}}>x\right) .
$$

So, we substitute (3.14) into (3.13) to obtain that

$$
\begin{aligned}
P\left(\widetilde{D}_{r}\left(t_{0}\right)>x\right) & \gtrsim \sum_{i=1}^{\infty} P\left(\left(X_{i}+Y_{i} e^{-r T_{i}}\right) e^{-r \tau_{i}} \mathbf{1}_{\left\{\tau_{i} \leq t_{0}\right\}}>x\right) \\
& =\int_{0-}^{t_{0}} \bar{U}\left(x e^{r t}\right) d E N(t) .
\end{aligned}
$$

Under condition 1 of Theorem 2.1, we show from (3.6) and (3.15) that for all large $x \geq D$,

$$
\text { (3.16) } \begin{aligned}
& \psi_{r}(x) \\
\geq & P\left(\widetilde{D}_{r}\left(t_{0}\right)>x+\widetilde{C}+\widetilde{B}\right) \\
\geq & \int_{0-}^{\infty} \int_{0-}^{t_{0}} \bar{U}\left((x+y) e^{r t}\right) d E N(t) P(\widetilde{C}+\widetilde{B} \in d y) \\
\sim & \int_{0-}^{t_{0}} \bar{U}\left(x e^{r t}\right) d E N(t) \\
= & \left(\int_{0-}^{\infty}-\int_{t_{0}}^{\infty}\right) \bar{U}\left(x e^{r t}\right) d E N(t) \\
\geq & (1-\varepsilon)\left(\int_{0-}^{\infty} \bar{F}\left(x e^{r t}\right) d E N(t)+\int_{0-}^{\infty} \int_{0-}^{\infty} \bar{G}\left(x e^{r(u+t)}\right) d H(u) d E N(t)\right),
\end{aligned}
$$

where the third step is due to $U \in \mathcal{C} \subset \mathcal{L}$ and the local uniformity of (1.4), and the last step is due to (3.12) and Lemma 3.3. Therefore, by (3.10), (3.16) and 
the arbitrariness of $\varepsilon>0$, we obtain that relation (1.5) holds under condition 1 of this theorem.

Under condition 2 of Theorem 2.1, we know from $U \in \mathcal{C}$ that for the given $\varepsilon>0$, there exists a number $\delta_{0}>0$ such that for all large $x$,

$$
\bar{U}\left(\left(1+\delta_{0}\right) x\right) \geq(1-\varepsilon) \bar{U}(x) .
$$

By (3.6), we see that for $\delta_{0}>0$ as above,

$$
\psi_{r}(x) \geq P\left(\widetilde{D}_{r}\left(t_{0}\right)-\widetilde{B}>\left(1+\delta_{0}\right) x\right)-P\left(\widetilde{C}>\delta_{0} x\right)=H_{5}-H_{6} .
$$

For $H_{5}$, arguing as (3.16) and using (3.17) leads to that for all large $x$,

$$
\begin{aligned}
H_{5} & \gtrsim \int_{0-}^{t_{0}} \bar{U}\left(\left(1+\delta_{0}\right) x e^{r t}\right) d E N(t) \\
& \geq(1-\varepsilon)\left(\int_{0-}^{\infty}-\int_{t_{0}}^{\infty}\right) \bar{U}\left(x e^{r t}\right) d E N(t) \\
& \geq(1-\varepsilon)^{2}\left(\int_{0-}^{\infty} \bar{F}\left(x e^{r t}\right) d E N(t)+\int_{0-}^{\infty} \int_{0-}^{\infty} \bar{G}\left(x e^{r(u+t)}\right) d H(u) d E N(t)\right),
\end{aligned}
$$

where the last step is due to (3.12) and Lemma 3.3. For $H_{6}$, by $(2.3), F \in \mathcal{C} \subset$ $\mathcal{D}, G \in \mathcal{C} \subset \mathcal{D}$ and Lemma 3.2(1), we have

$$
\begin{aligned}
H_{6} & =o(1)(\bar{F}(x)+\bar{G}(x)) \\
& =o(1)\left(P\left(X_{1} e^{-r \tau_{1}}>x\right)+P\left(Y_{1} e^{-r\left(\tau_{1}+T_{1}\right)}>x\right)\right) \\
& =o(1) \sum_{i=1}^{\infty}\left(P\left(X_{i} e^{-r \tau_{i}}>x\right)+P\left(Y_{i} e^{-r\left(\tau_{i}+T_{i}\right)}>x\right)\right) \\
& =o(1)\left(\int_{0-}^{\infty} \bar{F}\left(x e^{r t}\right) d E N(t)+\int_{0-}^{\infty} \int_{0-}^{\infty} \bar{G}\left(x e^{r(u+t)}\right) d H(u) d E N(t)\right) .
\end{aligned}
$$

Consequently, by (3.10), (3.18)-(3.20) and the arbitrariness of $\varepsilon>0$, we arrive at relation (1.5) under condition 2 of the theorem, and hence the proof is completed.

Acknowledgement. The authors would like to thank the anonymous referee for her/his constructive comments which greatly improved the quality and the presentation of this paper. The research was supported by the National Natural Science Foundation of China (NOs. 11326176, 71271042 and 71471090), the Natural Science Foundation of Jiangsu Higher Education Institutions of China (NOs. 13KJB110014 and 14KJB110014) and the Projects Funded by the Priority Academic Program Development of Jiangsu Higher Education Institutions and the Key Lab of Financial Engineering of Jiangsu Province. 


\section{References}

[1] N. H. Bingham, C. M. Goldie, and J. L. Teugels, Regular Variation, Cambridge University Press, Cambridge, 1987.

[2] Y. Chen and K. C. Yuen, Sums of pairwise quasi-asymptotically independent random variables with consistent variation, Stoch. Models 25 (2009), no. 1, 76-89.

[3] D. B. H. Cline and G. Samorodnitsky, Subexponentiality of the product of independent random variables, Stochastic Process. Appl. 49 (1994), no. 1, 75-98.

[4] P. Embrechts, C. Klüppelberg, and T. Mikosch, Modelling Extremal Events for Insurance and Finance, Springer, Berlin, 1997.

[5] Q. Gao and D. Bao, Asymptotic ruin probabilities in a generalized jump-diffusion risk model with constant force of interest, J. Korean Math. Soc. 51 (2014), no. 4, 735-749.

[6] Q. Gao and N. Jin, Randomly weighted sums of pairwise quasi upper-tail independent increments with application to risk theory, Comm. Statist. Theory Methods (2015), 10.1080/03610926.2013.851234.

[7] Q. Gao, N. Jin, and H. Shen, Asymptotic behavior of the finite-time ruin probability with pairwise quasi-asymptotically independent claims and constant interest force, Rocky Mountain J. Math. 44 (2014), no. 5, 1505-1528.

[8] Q. Gao and X. Liu, Uniform asymptotics for the finite-time ruin probability with upper tail asymptotically independent claims and constant force of interest, Statist. Probab. Lett. 83 (2013), no. 6, 1527-1538.

[9] Q. Gao and X. Yang, Asymptotic ruin probabilities in a generalized bidimensional risk model perturbed by diffusion with constant force of interest, J. Math. Anal. Appl. 419 (2014), no. 2, 1193-1213.

[10] Q. Gao and Y. Yang, Uniform asymptotics for the finite-time ruin probability in a general risk model with pairwise quasi-asymptotically independent claims and constant interest force, Bull. Korean Math. Soc. 50 (2013), no. 2, 611-626.

[11] J. Geluk and Q. Tang, Asymptotic tail probabilities of sums of dependent subexponential random variables, J. Theoret. Probab. 22 (2009), no. 4, 871-882.

[12] J. Li, On pairwise quasi-asymptotically independent random variables and their applications, Statist. Probab. Lett. 83 (2013), no. 9, 2081-2087.

[13] J. Li and R. Wu, The Gerber-Shiu discounted penalty function for a compound binomial risk model with by-claims, Acta Math. Appl. Sin. Engl. Ser. 31 (2015), no. 1, 181-190.

[14] X. Liu, Q. Gao, and Y. Wang, A note on a dependent risk model with constant interest rate, Statist. Probab. Lett. 82 (2012), no. 4, 707-712.

[15] H. Meng and G. Wang, On the expected discounted penalty function in a delayed-claims risk model, Acta Math. Appl. Sin. Engl. Ser. 28 (2012), no. 2, 215-224.

[16] Q. Tang, Asymptotic ruin probabilities of the renewal model with constant interest force and regular variation, Scand. Actuar. J. 2005 (2005), no. 1, 1-5.

[17] Q. Tang and G. Tsitsiashvili, Precise estimates for the ruin probability in finite horizon in a discrete-time model with heavy-tailed insurance and financial risks, Stochastic Process. Appl. 108 (2003), no. 2, 299-325.

[18] _ Randomly weighted sums of subexponential random variables with application to ruin theory, Extremes 6 (2003), no. 3, 171-188.

[19] D. Wang, C. Su, and C. Zeng, Uniform estimate for maximum of randomly weighted sums with applications to insurance risk theory, Sci. China Ser. A 48 (2005), no. 10, 1379-1394.

[20] K. Wang, Y. Wang, and Q. Gao, Uniform asymptotics for the finite-time ruin probability of a dependent risk model with a constant interest rate, Methodol. Comput. Appl. Probab. 15 (2013), no. 1, 109-124.

[21] Y. Wang and D. Cheng, Basic renewal theorems for a random walk with widely dependent increments and their applications, J. Math. Anal. Appl. 384 (2011), no. 2, 597-606. 
[22] H. R. Waters and A. Papatriandafylou, Ruin probabilities allowing for delay in claims settlement, Insurance Math. Econom. 4 (1985), no. 2, 113-122.

[23] Y. Xiao and J. Guo, The compound binomial risk model with time-correlated claims, Insurance Math. Econom. 41 (2007), no. 1, 124-133.

[24] H. Xie and W. Zou, Expected present value of total dividends in a delayed claims risk model under stochastic interest rates, Insurance Math. Econom. 46 (2010), no. 2, 415422.

[25] _ On the expected discounted penalty function for the compound Poisson risk model with delayed claims, J. Comput. Appl. Math. 235 (2011), no. 8, 2392-2404.

[26] K. C. Yuen and J. Guo, Ruin probabilities for time-correlated claims in the compound binomial model, Insurance Math. Econom. 29 (2001), no. 1, 47-57.

[27] K. C. Yuen, J. Guo, and K. W. Ng, On ultimate ruin in a delayed-claims risk model, J. Appl. Probab. 42 (2005), no. 1, 163-174.

[28] W. Zou and H. Xie, On the probability of ruin in a continuous risk model with delayed claims, J. Korean Math. Soc. 50 (2013), no. 1, 111-125.

Qingwu Gao

International Center of Management Science and Engineering

School of Management and Engineering

NANJING UNIVERSITY

NANJING 210093, P. R. ChinA

AND

SCHOOl of SCIENCE

NANJing Audit University

NANJing 211815, P. R. China

E-mail address: qwgao0123@gmail.com, qwgao@aliyun.com

ERLi ZHANG

Department of Computer Science

Zhengzhou Institute of Finance and Economics

Zhengzhou 450000, P. R. China

E-mail address: isszel@163.com

NA JiN

Jiangsu Price Institute

Jiangsu Price Bureau

NANJING 210024, P. R. China

E-mail address: kingna3394@163.com 